\title{
Impact of Age on Color Choice/Preference
}

\section{Anwesha Sengupta, Ayindrela Halder, Sayani Biswas, Shinjini Saha, Tinni Dutta*}

\author{
Department of Psychology, Asutosh College, University of Calcutta, Kolkata, India \\ *Corresponding author details: Dr. Tinni Dutta, tinnid@yahoo.com
}

\begin{abstract}
Color preference is an integral aspect of human behaviour, and its importance is quite noticeable. This study aims at the identification of color preferences among different ages. This will help to better understand the implication of colors in color therapy for dealing with real life practical situations. In this study, two groups of 20 people each, ranging from 18 to 35 years of age and 55 to 70 years of age were chosen from both the sexes. The color preference among the individuals were evaluated based on their response for the most and least preferred colors, and the results analysed according to the mean, median, standard deviation, and t-test. It is found that there lies a significant difference between one's age and color preference, although blue was the most preferred color among both the groups.
\end{abstract}

Keywords: color preference; age difference; color therapy

\section{INTRODUCTION}

The Webster dictionary defines colors as a phenomenon of light (such as red, brown, pink or gray) or visual representation that enables one to differentiate otherwise identical objects. It's the characteristic of visual perception described through color categories with names as red, blue, orange, yellow, green or purple.

Where there are hundreds of theories on color vision and understanding the neuroscience and physics of color, the present study focuses on the psychology of preferring one color over the other. The motive of this study is to gain a better understanding of the 'why' of a color. The psychology of color preference has long been studied since the late nineteenth century. Although little is known about exactly which and why certain colors are liked over the other, given that color selection is such a pervasive every day activity. So the aim of this study topic rests on digging a little deeper on the topic. Carl Jung is most prominently associated with the pioneering stages of color psychology. Jung was most interested in colors' properties and meanings, as well as in art's potential as a tool for psychotherapy. His studies in and writings on color symbolism cover a broad range of topics, from mandalas to the works of Picasso to the nearuniversal sovereignty of the color gold.

The general model of color psychology relies on six basic principles:

1. Color can carry a specific meaning.

2. Color meaning is either based in learned meaning or biologically innate meaning.

3. The perception of a color causes evaluation automatically by the person perceiving.

4. The evaluation process forces color-motivated behavior.

5. Color usually exerts its influence automatically.

6. Color meaning and effect has to do with context as well.

The existing literature on this topic states that humans can distinguish about 10 million colors. At the retinal level, three different types of cones are sensitive to different ranges of the visible spectrum, i.e., Long (L), Medium (M) and Short (S) cones are sensitive mainly to the reddish, greenish and bluish thirds of the spectrum respectively.

Hulbert and Ling proposed that color preferences are based on hard wired cone opponent mechanisms in the human visual system that arose from evolutionary selection. According to the ecological valence theory, color preferences stem from color-relevant experiences during an individual's life. Evolutionary biological accounts predict universal and sex specific patterns in color preference, accounts based on associative learning suggests preferences arise from individual, color relevant experiences with objects or concepts.

It is to be noted that favorite colors in general may not be the same colors as favourite for specific objects.

A review of color studies by Eysenck, in the early 1940s notes a relationship between gender and color. He found that women preferred yellow over orange whereas, men preferred the opposite. A later study conducted by Birren (1952) reinforced Eysenck's study further. Dorcus in the early eighteenth century found yellow had a higher affective value for the men than women and St. George (1938) maintained that blue for men stands out far more than for women. These studies explained the gender difference in color preferences.

Cultural background has been shown to have a strong influence on color associations \& preference. Studies have shown that while people from the same region regardless of race will have the same color preferences, common associations connecting a color to a particular emotion may differ cross-culturally. For instance, one study that examined color associations with emotion with participants from Germany, Mexico, Poland, Russia, and the United States found that in all nations the color red was associated with anger and was perceived as strong and active. However, only Poles associated purple with both anger and jealousy while Germans associated jealousy with yellow, highlighting how the influence of different cultures can potentially change perceptions of color and its relationship to emotion. 
Aesthetic ratings are often used to assess color preferences. In a forced choice, paired comparison task, participants choose the color they like more than another color presented simultaneously. The least chosen color is assumed to represent the most disliked color even though participants did not make this judgment directly. Thus the results from studies on color preferences using this method of testing or experimentation indicate that disliked colors seem to lie in the range of dull and dark yellows and greens.

Multiple studies that are conducted revealed that the general mode of peoples color preference is: Blue, Red, Green, Purple, Orange, and Yellow $\mathrm{Cu}$, Luo, Woodcock and Wright, 2004; Hurlbert and Ling 2007, Palmer and Schloss, 2010).

According to Elliot and Maire (2004) colors can affect people's emotions, cognition, actions and also physiological processes. According to Huchendorf (2007), color has been found to increase a person's arousal. McManus, Jones and Cottrell (1981) stated that color preference increases with greater brightness. But according to Taylor, Clifford and Franklin (2012) color preference decreases with greater brightness. Schloss, Strauss and Palmer (2013) stated that people prefer color with high saturation among finite color samples.

According to Birren(2006), colors have many emotional impacts for instance, bright and low saturation creates soft feelings whereas, dim and high saturation creates hard feelings. Birren also asserted that warm colors like red and yellow, increase arousal more than cool colors such as blue and green. He also stated that outwardly integrated people prefer warm colors whereas inwardly integrated people like cool colors and are not enthusiastic about any colors.

Manuela (2000) researched whether color preference changes with ageing,and found that color preference would have a change in adolescence. Blue became a popular color during that time. But as the age increases, love for blue decreases and love for green and red increases.

Dittmar (2001) also stated that old people started liking green and red. Burkitt (2003) said that children choose warm colors over cold and dark colors. Buchanan (1984) studied college students and found that people's favourite color varies based on different objects and normally their favourite colors are different from object colors.

The purpose of the present research paper is to study the impact of age on color preference. Thus, generating an alternative hypothesis stating that age influences one's sense color preference; and a null hypothesis stating age has no impact on the preference of colors. In other words, to investigate whether there is a significant difference in the calculated statistical values, of different age groups, that support either of the hypotheses mentioned above.

\section{METHOD}

\section{PROCEDURE}

Rapport was established with each participant. Four primary colors namely Red, Blue, Green, Yellow were chosen, so that no confusion occurs. From these four colors, 6 pairs of colors were made (Red-Blue, Blue-Green, Green-Yellow, Red-Green, Yellow-Blue, and Red-Yellow) and they were presented to the participants over the phone due to the pandemic situation. The participants had to choose any one color from each pair which they preferred the most. This way, 40 data was collected from each age group (18-25 and 60-75) and necessary statistical calculations (mean, median, mode, standard deviation, t-test) were done for further discussion.

Here, a scoring system has been established, where the most preferred color has been attributed a score of 100 and the less preferred color has been attributed a score of 0 .

\section{SAMPLING}

Sampling was done on the basis of randomisation method for the fair representation of the population. Two groups were selected namely, group one 18-35 of age, and group two 55-70 of age. 20 people (both male and female) from each group were selected.

\section{CALCULATION}

Statistical measures like mean, median, standard deviation and t-test are done on the obtained data. The mean and median are the measures of central tendency. They give a summary of the data obtained from the participants. The standard deviation is a measure variability which describes how varied one's data is on a normal distribution. And lastly, the t-test is an inferential statistics used to testify the hypothesis for any study/research. It is used to determine whether the calculated means of any two groups at hand are significant or not, using degrees of freedom. These statistical calculations will help in drawing a proper result and interpretation for the present research.

\section{RESULTS}

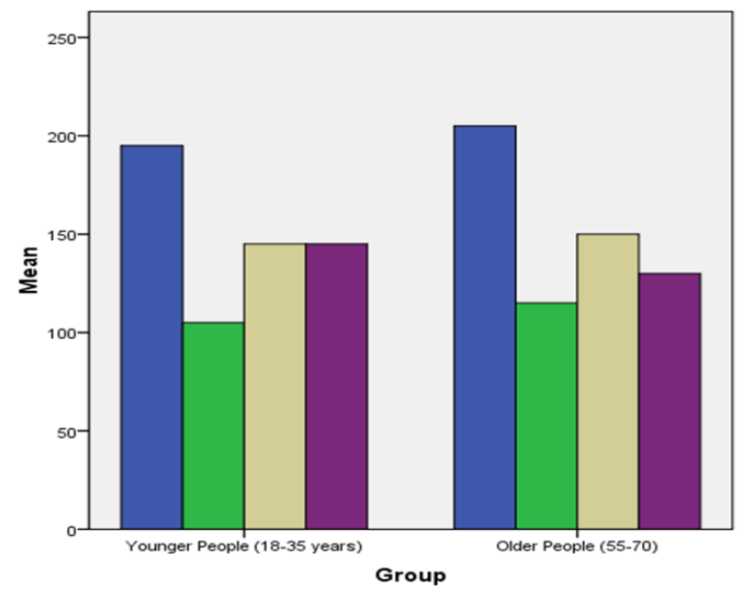

Here, is the graphical representation of the mean of all the colors between two groups. It is clearly seen that the mean of blue is distinctly higher than the rest of the colors in both the groups.

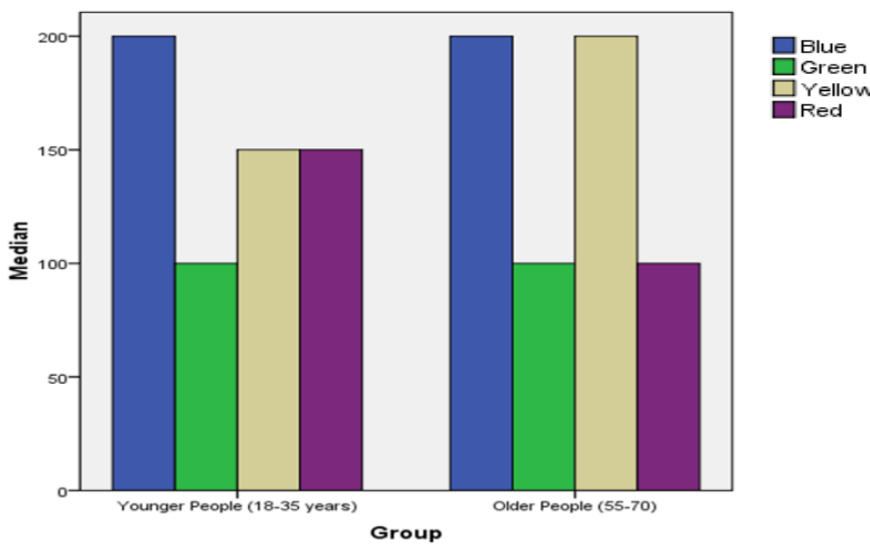

Here, is the graphical representation of the median of all the colors between two groups. It is clearly seen that the mean of blue color is distinctly higher than the rest 
of the colors in the first group i.e., younger people. But in the group of older people the median of both yellow and blue are same and higher than the rest.

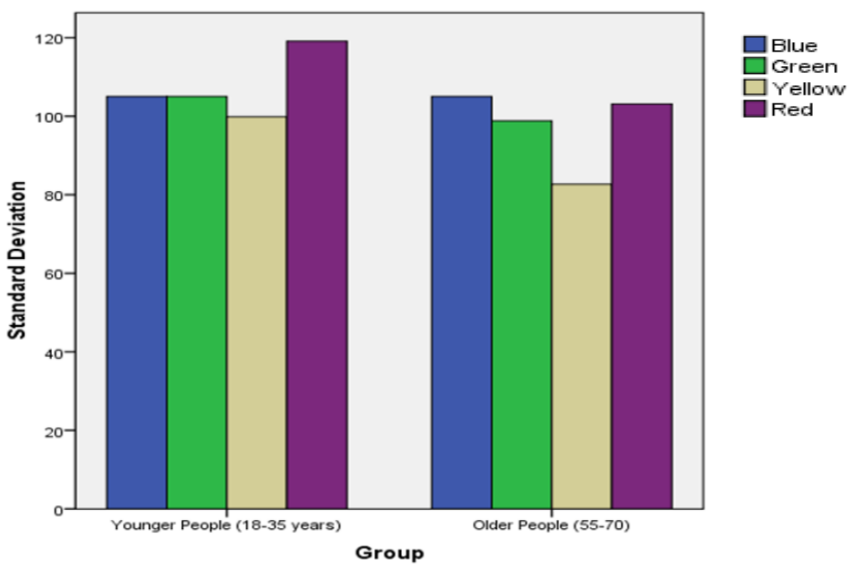

Here, is the graphical representation of the standard deviation of all the colors between two groups. It is seen that the standard deviation of red is distinctly higher in the group of young adults. In the elderly group the standard deviation of blue and red are highest and doesn't have much of a difference with green.

\section{DISCUSSION}

As per the obtained result, it is seen that there is a significant difference between one's age and color preference. So, the alternative hypothesis is accepted and the null hypothesis is rejected.

The calculated values of mean, median, mode, standard deviation and t-test support the above mentioned hypothesis. Hence, indicating that age leaves a certain amount of influence on the choice of colors. That is, with one's advancing age one's color taste and preference keeps changing. In the present study it was found out that participants falling between the age group of 18 to 35 years mostly preferred blue, yellow and purple, blue being the most preferred color following which yellow and purple both are second in the list of preferences ; whereas participants in their 50s -70s mostly preferred blue and yellow. It can be seen that though the color blue remains common in both the groups, the preference for blue among the older participants is higher than that in the younger participants. The color blue is interpreted as a sign of maturity, people keeping blue as their highest preferred color are usually introverted and keep their realisations to themselves, they are also very aware of their environment. The results seem quite in synchrony with the existing literature on color preferences.

Color Therapy is a method of treatment that uses the visible spectrum (colors) of electromagnetic radiations (wavelength 7700-3900AU) and invisible spectrum (infrared and UV) to cure diseases. As per the obtained results, it is seen that there is an impact of age on color preference. So we can use color therapy to cure diseases. Colors are beneficial for every man in many respects. Studies demonstrated that color has psychological as well as physiological implementation.

Psychological implementation of Red is vitality, strength, sexuality, will power and alertness.

Psychological implementation of Blue is will power and communication, management of migraine due to psychological factors.
Psychological implementation of Yellow is intellect, judgement, mental stability, concentration and that of Green is purity and harmony. Color therapy also has an effect on cancer, peptic ulcer, skin diseases, color blindness, jaundice, etc. Color therapy has wide scope in the near future.

\section{LIMITATIONS}

Like any other study/experiment this study too had its setbacks and limitations that, if controlled in the future may produce even better results. As the sample for the present study was too small, the generalization of the results on an entire population remains restricted. Due to the outbreak of the Covid-19 pandemic, the data was collected over phone calls and messages which didn't allow the participants to know the exact hue, lightness and saturation of the colors chosen for the study, but some researchers have found attributes of color influence the color preference $(\mathrm{Ou}$, et $\mathrm{al}, 2004)$, it is mostly subjected to their own imagined shade, as using visual cue would have been more objective. These few limitations if handled well in the future while replicating the study may bring out enhanced results.

\section{EXPECTATIONS}

1. Previous researches have worked only with primary colors. But in our daily life we not only come across the primary colors but also many complex colors, so future studies may work with different complex colors.

2. Most of the previous researchers did not work with the influence made by the context, so future studies may need to add context as a factor.

\section{CONCLUSION}

The purpose of this paper was to study the impact of color preference on one's age. Color is an important part of our life so it has an influence on every person. From the obtained result it was noted that in the present study both the younger and older groups of participants preferred the color blue over the rest of the colors. Color preference is just a branch of study of color. Future studies should solve existing problems and apply these researches to other fields.

\section{REFERENCES}

[1] Jonauskaite, D., Mohr, C., Antonietti, J.P., Spiers, P.M., Althaus, B., Anil, S., Dael, N., (2016). Most and Least Preferred Colors Differ According to Object Context: New Insights from an Unrestricted Color Range. Retrieved from https://journals.plos.org/plosone/article?id=10.13 71/journal.pone.0152194\#abstract0

[2] Miao, R., O. Color Preference. Retrieved from https://www.cs.bham.ac.uk/ rjh/courses/Research TopicsInHCI/2016-17/Submissions/miaoruijing.pdf

[3] Eysenck, H. J. (1941). A critical and experimental study of color preferences. The American Journal of Psychology, 54, 385-394. https://doi.org/10.2307/1417683

[4] Palmer, S. E., Schloss, K. B., Sammartino, J., (2013). Visual Aesthetics and Human Preference. Retrieved from https://palmerlab.berkeley.edu/pdf/PalmerSchlos sSammartino(2013)AR.pdf

[5] Palmer, S. E., Schloss, K. B., (2010). An ecological valence theory of human color preference. Retrieved from:https://www.ncbi.nlm.nih.gov/pmc/articles/P MC2889342/

[6] Strauss, E. D., Schloss, K. B., Palmer, S. E., (2013). Color preferences change after experience with liked/disliked color objects. Retrieved from 
[7] https://link.springer.com/article/10.3758/s13423013-0423-2

[8] McManus, I. C., Jones, A. L., \& Cottrell, J. (1981). The aesthetics of color. Perception, 10(6), 651-666. https://doi.org/10.1068/p100651

[9] Taylor, C., Clifford, A., \& Franklin, A. (2012, November 12). Color Preferences Are Not Universal. Journal of Experimental Psychology: General. Advance online publication. doi:10.1037/a0030273

[10] Dittmar, M. (2001). Changing Color Preferences with Ageing: A Comparative Study on Younger and Older Native Germans Aged 19-90 Years. Gerontology, 47(4), 219-226. doi:10.1159/000052802

[11] Holmes, C. B., \& Buchanan, J. A. (1984). Color preference as a function of the object described. Bulletin of the Psychonomic Society, 22(5), 423-425. https://doi.org/10.3758/BF03333865

[12] Silver, N. C., \& Ferrante, R. A. (1995). Sex Differences in Color Preferences among An Elderly Sample. Perceptual and Motor Skills, 80(3), 920-922. doi:10.2466/pms.1995.80.3.920

[13] Birren, F. (1961). Color psychology and color therapy: a factual study of the influence of color on human life. [Rev. ed.] Secaucus, N.J.: Citadel Press

[14] Hurlbert, A. C., \& Ling, Y. (2007). Biological components of sex differences in color preference. Current Biology, 17(16), R623-R625. doi:10.1016/j.cub.2007.06.022

[15] Ou, L.-C., Luo, M. R., Woodcock, A., \& Wright, A. (2004). A study of color emotion and color preference. Part I: Color emotions for single colors. Color Research \& Application, 29(3), 232-240. doi:10.1002/col.20010

[16] Kurt, S., Osueke, K. K., (2014). The effects of color on the moods of college students. Retrieved from https://journals.sagepub.com/doi/full/10.1177/2 158244014525423

[17] Huchendorf, Lynnay \& Cary, Melanie. (2007). The Effects of Color on Memory.

[18] Briggs, D., \& Westland, S. (2014). Itten, Johannes. Encyclopedia of Color Science and Technology, 1-3. doi:10.1007/978-3-642-27851-8_340-1

[19] Greene, T. C., Bell, P. A., Boyer, W. A., (1983). Coloring the environment: Hue, arousal and boredom. Retrieved from https://link.springer.com/content/pdf/10.3758/B F03334701.pdf 